$12-15-2012$

\title{
An Elastica Model of the Buckling of a Nanoscale Sheet Perpendicular to a Rigid Substrate
}

\author{
Shawn D. Ryan \\ Cleveland State University, s.d.ryan@csuohio.edu \\ Dmitry Golovaty \\ University of Akron \\ Patrick Wilber \\ University of Akron, jw50@uakron.edu
}

Follow this and additional works at: https://engagedscholarship.csuohio.edu/scimath_facpub

Part of the Mathematics Commons

How does access to this work benefit you? Let us know!

\section{Repository Citation}

Ryan, Shawn D.; Golovaty, Dmitry; and Wilber, Patrick, "An Elastica Model of the Buckling of a Nanoscale Sheet Perpendicular to a Rigid Substrate" (2012). Mathematics Faculty Publications. 310.

https://engagedscholarship.csuohio.edu/scimath_facpub/310

This Article is brought to you for free and open access by the Mathematics and Statistics Department at EngagedScholarship@CSU. It has been accepted for inclusion in Mathematics Faculty Publications by an authorized administrator of EngagedScholarship@CSU. For more information, please contact library.es@csuohio.edu. 


\title{
An elastica model of the buckling of a nanoscale sheet perpendicular to a rigid substrate
}

\author{
Shawn D. Ryan ${ }^{\text {a }}$, Dmitry Golovaty ${ }^{\mathrm{b}}$, J. Patrick Wilber ${ }^{\mathrm{b}}$ \\ ${ }^{a}$ Department of Mathematics, Pennsylvania State University, University Park, PA 16802, United States \\ ${ }^{\mathrm{b}}$ Department of Mathematics, University of Akron, Akron, $\mathrm{OH} 44325-4002$, United States
}

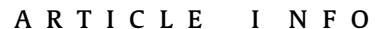

Article history:

Received 12 July 2011

Received in revised form 13 July 2012

Available online 20 August 2012

Keywords:

Van der Waals forces

Elasticity

Nanomechanics

Elastica

Buckling

\section{Introduction}

We study a novel variation on the classical problem of the buckling of an elastica. The elastica models the cross-section of a nanoscale sheet that interacts with a rigid substrate by van der Waals forces. See Fig. 1. The interaction is modeled as a body force of Lennard-Jones type. Specifically, the van der Waals interaction between a point on the sheet and the substrate is repulsive when the point is sufficiently close to the substrate, weakly attractive over some intermediate distance, and then essentially zero when the point on the elastica is far from the substrate. In our problem, the sheet is perpendicular to the substrate prior to buckling. A load is applied to the edge of the sheet further from the substrate. That the forces on the sheet sum to zero sets the spacing between the substrate and the edge of the sheet nearest the substrate. At this spacing the load applied to the far edge of the sheet balances the van der Waals interaction between the substrate and points on the sheet. We study how the sheet buckles from this configuration as the applied load varies.

Our study is motivated in part by an interest in the mechanical properties of graphene sheets. A graphene sheet is a singleatom-thick layer of carbon atoms in which each atom is bonded to its three nearest neighbors to form a hexagonal lattice. Stacked graphene sheets are the building blocks of graphite. Within the last decade, mechanical and chemical methods have been developed for isolating individual graphene layers (Novoselov et al., 2004,
2005; Schniepp et al., 2006). Numerous studies have demonstrated that graphene sheets have remarkable mechanical properties (Booth et al., 2008; Geim, 2009; Lee et al., 2008; Suk et al., 2010).

Because of their mechanical as well as electrical properties, graphene sheets may be useful components in a variety of potential nanoscale devices (Bunch et al., 2007; Liao and Koide, 2011; Wu et al., 2010). The successful development of such devices may entail the precise mechanical manipulation of individual graphene sheets. Our problem can be viewed as an idealized continuum model of the fabrication or operation of a nanoscale device in which a graphene sheet is positioned perpendicularly against a rigid substrate. It may be important to predict how close to the substrate the sheet can be positioned before buckling into a shape that is no longer flat and perpendicular to the substrate. We note, however, that research has shown that the edges of freely suspended graphene sheets may be wavy or rippled (Lu and Huang, 2010; Reddy et al., 2009, 2011), a geometry that is not described in our idealized continuum model. Also, our continuum model ignores thermal fluctuations of the atoms of which a graphene sheet is composed.

Although primarily motivated by an interest in the mechanics of graphene, our study fits in with a larger effort to understand the mechanics of any deformable nanoscale sheet, tube, filament, etc. interacting with nearby rigid structures. Areas where such problems arise include the operation of MEMS (Jia et al., 2011; Zhao et al., 2003), the mechanics of polymers (Aliee and Najafi, 2008; Smith et al., 2001; Rief et al., 1999; Purohit et al., 2003), and the adhesion of fibers and hairs (Autumn et al., 2002; Bico et al., 2004; Majidi et al., 2005). Essential for understanding 


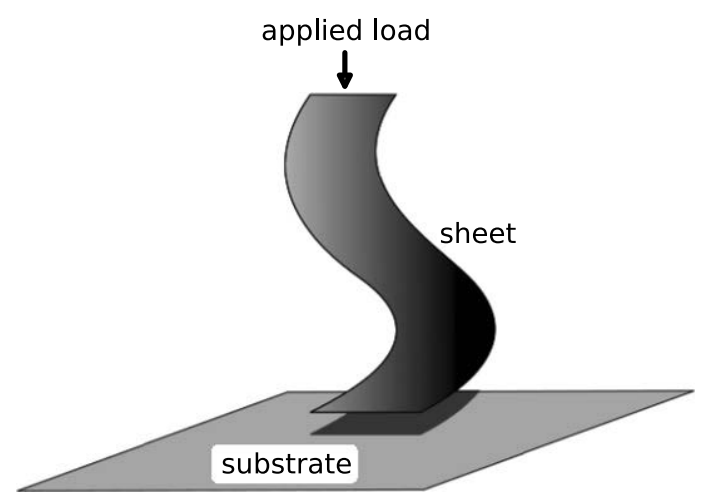

Fig. 1. Nanoscale sheet deforming above a rigid substrate.

mechanics at this length scale is predicting how bodies deform in response to the interplay between elasticity and intermolecular forces (Attard and Parker, 1992; Evans and Lauga, 2009; Oyharcabal and Frisch, 2005). The straightforward geometry of our problem makes it a natural one to consider in this context.

The existing work on buckling of graphene sheets ( $\mathrm{Li}$ and Chou, 2003; Lu and Huang, 2009; Pradhan and Murmu, 2009, 2010; Sakhaee-Pour, 2009; Wilber et al., 2007a) is based on various approaches including atomistic simulations, continuum beam theory, and non-local elasticity. None of these papers considers the buckling of graphene interacting with a rigid substrate. See the introduction in Wilber (2010) for more detailed comments. This paper is complementary to Wilber (2010), which uses a similar model to explore a buckling problem in an alternative geometry in which the sheet is parallel to the substrate prior to buckling (hence yielding a variation on the classical problem of an elastica on a nonlinearly elastic foundation).

The same continuum model we present here is used in Oyharcabal and Frisch (2005) to study the adhesion by van der Waals forces of an elastica to a substrate. The authors study numerically how the elastica, initially parallel to the substrate at the equilibrium spacing, peels off the substrate under an applied load at one end. A closely related study is Strus et al. (2008), in which the authors use the same model to investigate the peeling of a carbon nanotube from a substrate. In another related paper, Evans and Lauga (2009), the authors use linear beam theory to study how two or several sheets interacting by a van der Waalslike force adhere to one another.

In Strus and Raman (2009), the model from Oyharcabal and Frisch (2005) is adapted to predict the deformation of carbon nanotubes used as probe tips in atomic force microscopy. The boundary-value problem we formulate in Section 2 is similar to the 'perfect slip' case studied in Strus and Raman (2009), except that we use a pinned condition at the top of the sheet while the authors in Strus and Raman (2009) use a clamped condition at the top of the tube. In Strus and Raman (2009), the authors use the model to study post-buckling behavior by numerically continuing the solution off the trivial branch from what we call the pulling bifurcation point. They plot the applied load versus the separation of the top of the nanotube from the substrate to describe how the nanotube transitions between different configurations as the tube contacts the substrate. Here we do not consider post-buckling behavior except locally near bifurcation points. See the remark at the end of Section 3.4 below for an additional comment on how our results compare with those of Strus and Raman (2009).

We model the cross-section of the sheet as an elastica. We use a variational approach, in which the energy has two parts, the bending energy of the elastica and the energy from the interaction of the elastica with the substrate by van der Waals forces. For the buckling problem described above, we identify a trivial branch, which corresponds to the elastica remaining straight and perpendicular to the substrate. Upon linearizing about this trivial branch, we obtain a classical self-adjoint eigenvalue problem. A mix of analysis and numerical computation is used to study this eigenvalue problem and determine the stability and bifurcations of solutions along the trivial branch.

To summarize our main results, we imagine an experiment that starts with the bottom edge of the sheet far from the substrate. The van der Waals force between points on the substrate and each point on the sheet is attractive, and the applied load pulls the sheet away from the substrate to balance the van der Waals force pulling the sheet toward the substrate. At this spacing the straight configuration is stable. If the applied load is slowly increased, equilibrium is maintained by allowing the spacing between the bottom edge of the sheet and the substrate to increase. If this process is continued, the bottom edge of the sheet approaches a spacing at which the total attractive van der Waals force on the sheet is at a maximum. A further increase in the applied load cannot be balanced by the attractive force of the substrate. The straight configuration loses stability, not through buckling, but rather because after a small displacement away from the substrate, the sheet keeps moving away from the substrate.

On the other hand, if at the initial stable spacing, the applied load is slowly decreased, equilibrium can be maintained by allowing the spacing between the bottom edge of the sheet and the substrate to decrease. If this process is continued, the bottom edge of the sheet approaches the substrate and the van der Waals force on points near the bottom of the sheet becomes repulsive, although the total van der Waals force on the sheet is still attractive. At some critical applied load, the straight configuration loses stability and the sheet buckles into a configuration in which the edge of the sheet closest to the substrate kicks out to one side. See Fig. 7. We show that at this critical load the total van der Waals force on the sheet is attractive, so the applied load pulls the sheet away from the substrate.

This thought experiment summarizes our results on how the straight configuration of the sheet loses stability. We show that all additional bifurcation points on the trivial branch occur when the total van der Waals force on the sheet is repulsive, which means the applied load is pushing the sheet toward the substrate. There are a countable number of these additional bifurcation points that lie on an unstable section of the trivial branch.

We also formulate the boundary-layer problem that arises if the length of the sheet is large compared to the characteristic length over which the van der Waals interaction is significant. After introducing an appropriate small parameter, we perform a straightforward but illuminating bifurcation analysis of the inner and outer problems. The first two bifurcations described above-the sheet continuing to move away from the substrate after a small displacement, and the sheet kicking out to one side-arise in the inner problem. The countable number of bifurcation points that occur when the applied load is pushing the sheet into the substrate arises in the outer problem, which at first order has the form of a classical Euler buckling problem.

In the next section, we present the variational formulation of our problem and identify the trivial branch for our bifurcation analysis. In Section 3, by studying the minimization of the total energy we demonstrate the stability or instability along different parts of the trivial branch. In Section 3.3, bifurcation points are identified and the stability analysis is completed. Section 4 contains a bifurcation analysis for the limiting case of very long sheets. An Appendix that follows provides further details on the analysis of the eigenvalue problem studied in Sections 3.3 and 4 . 


\section{Formulation of the model}

We use continuum mechanics to model a nanoscale sheet interacting with a rigid substrate. To describe the geometry of our problem, we let $\{\mathbf{i}, \mathbf{j}, \mathbf{k}\}$ denote a right-handed orthonormal basis for $\mathbb{R}^{3}$. The rigid substrate is parallel to the ik-plane, and $\mathbf{j}$ points away from the substrate. See Fig. 2. We assume the deformation of the sheet is the same in every cross-section defined by a plane perpendicular to $\mathbf{k}$, and hence the configuration of the sheet is determined by the configuration of a typical cross-section. A cross-section is described by a curve $[0, L] \ni s \mapsto \mathbf{r}(s)$ in the ij-plane. Because we are modeling a graphene sheet, which is only a single layer of atoms thick, we do not need any additional kinematic variables to track points off of the 'centerline' described by $\mathbf{r}$ and we assume the sheet is unshearable.

We assume the sheet is perpendicular to the substrate prior to buckling. We shall refer to the edges closest to and farthest from the substrate as the bottom and top of the sheet. The positions of the bottom and the top of the sheet correspond to $s=0$ and $s=L$. We set $y_{0}=\mathbf{r}(0) \cdot \mathbf{j}$, which is the distance from the bottom of the sheet to the substrate. See Fig. 2. We assume that a constant load $v \mathbf{j}$ is applied to the top of the sheet. Hence $v>0$ corresponds to pulling the sheet away from the substrate and $v<0$ corresponds to pushing the sheet toward the substrate.

We use a variational approach to derive governing equations. It is convenient to introduce components by writing

$\mathbf{r}(s)=x(s) \mathbf{i}+y(s) \mathbf{j}$,

from which we see that $y_{0}=y(0)$. We assume that the sheet is inextensible, so that $\left|\mathbf{r}^{\prime}(s)\right|=1$ for all $s \in[0, L]$. It follows that $L$ is the length of the sheet and that there is a function $\theta$ such that

$\mathbf{r}^{\prime}(s)=\cos (\theta(s)) \mathbf{i}+\sin (\theta(s)) \mathbf{j}$.

Note that $\theta(s)$ is the angle between $\mathbf{i}$ and the tangent line at $\mathbf{r}(s)$ and that $\theta^{\prime}$ is the curvature of $\mathbf{r}$. The pair $y_{0}$ and $\theta$ determine the configuration of the sheet to within a rigid translation parallel to the substrate.

The potential energy per unit width of a sheet with configuration $\left(\theta, y_{0}\right)$ interacting with the substrate is

$$
\begin{aligned}
E\left[\theta, y_{0}\right]= & \int_{0}^{L} \frac{\beta}{2} \theta^{\prime}(s)^{2} d s+\int_{0}^{L} F\left(y_{0}+\int_{0}^{s} \sin \theta(\xi) d \xi\right) d s \\
& -v\left(y_{0}+\int_{0}^{L} \sin \theta(s) d s\right) .
\end{aligned}
$$

The first term in (2.3) is the bending energy stored in the sheet. Because we assume the sheet is inextensible and unshearable,

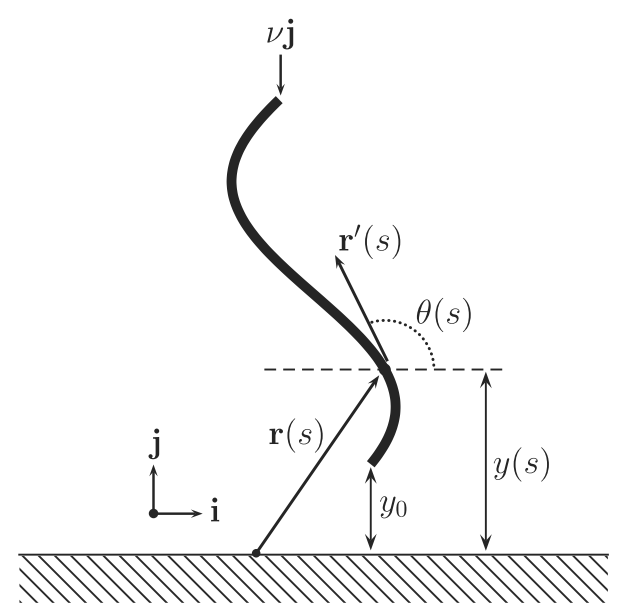

Fig. 2. Cross-section of the sheet deforming above the rigid substrate. energy is stored in the sheet only by bending. These assumptions along with the assumption that the stored-energy is a constant times the curvature squared are the classical elastica theory. (For further background on the potential energy for the elastica, see Caflisch and Maddocks (1984) as well as Antman (2005), Chapters IV,VII.) The constant $\beta$ in the first term is the bending stiffness of the sheet. Values from the literature on the continuum modeling of graphene and carbon nanotubes suggest that one can choose $\beta$ in a range between .13 and .2 nN nm (Kim and Castro Neto, 2008; Robertson et al., 1992; Sabio et al., 2008; Tersoff, 1992; Tu and Ou-Yang, 2002; Yakobson et al., 1996). To make explicit computations below, we take $\beta=0.14 \mathrm{nN} \mathrm{nm}$. The second term in (2.3) is a continuum approximation of the potential energy of the van der Waals interaction between the sheet and the substrate. The function $F$ in the second term is the energy per unit area of the van der Waals interaction between the substrate and the material point $s$. Because $y(s)=y_{0}+\int_{0}^{s} \sin \theta(\xi) d \xi$, we assume that $F$ depends just on the distance between the point $s$ and the substrate. We defer until later the derivation of the exact form of $F$. The third term in (2.3) is the work done by the applied load.

As noted above, a graphene sheet is only a single-layer of atoms thick. Its resistance to bending arises because bending the sheet requires changing the bond angles between carbon atoms. In classical linear shell theory, resistance to bending arises because bending the shell requires stretching or compressing material off the center surface of the shell. Hence in the classical theory, the bending modulus is related to the thickness of the shell and Youngs modulus of the material. In our model, by contrast, we do not need to assign a thickness to the sheet. See Peng et al. (2008) for an interesting discussion of this issue in the context of carbon nanotubes.

We obtain the equilibrium equations for our problem by seeking pairs $\left(\theta, y_{0}\right)$ at which $(2.3)$ is stationary. First we compute

$\left.\frac{\partial}{\partial \epsilon} E\left[\theta, y_{0}+\epsilon y_{1}\right]\right|_{\epsilon=0}=y_{1} \int_{0}^{L} F^{\prime}\left(y_{0}+\int_{0}^{s} \sin \theta(\xi) d \xi\right) d s-v y_{1}$,

which must vanish for all $y_{1}$, so that

$v=\int_{0}^{L} F^{\prime}\left(y_{0}+\int_{0}^{s} \sin \theta(\xi) d \xi\right) d s$.

We define $N$ on $[0, L]$ by

$N(s)=\int_{0}^{s} F^{\prime}\left(y_{0}+\int_{0}^{\xi} \sin \theta(\gamma) d \gamma\right) d \xi$,

with which (2.5) can be written as $N(L)=v$.

Next we compute

$$
\begin{aligned}
& \left.\frac{\partial}{\partial \epsilon} E\left[\theta+\epsilon \theta_{1}, y_{0}\right]\right|_{\epsilon=0} \\
& =\int_{0}^{L} \beta \theta^{\prime}(s) \theta_{1}^{\prime}(s) d s+\int_{0}^{L}\left[F^{\prime}\left(y_{0}+\int_{0}^{s} \sin \theta(\xi) d \xi\right)\right. \\
& \left.\quad \times \int_{0}^{s} \theta_{1}(\xi) \cos \theta(\xi) d \xi\right] d s-v \int_{0}^{L} \theta_{1}(s) \cos \theta(s) d s
\end{aligned}
$$

where $\theta_{1}$ is arbitrary. Noting that $N^{\prime}(s)=F^{\prime}\left(y_{0}+\int_{0}^{s} \sin \theta(\xi) d \xi\right)$, we integrate the first two terms in the right-hand side of (2.7) by parts, which gives

$$
\begin{aligned}
\left.\frac{\partial}{\partial \epsilon} E\left[\theta+\epsilon \theta_{1}, y_{0}\right]\right|_{\epsilon=0}= & -\int_{0}^{L}\left(\beta \theta^{\prime \prime}(s)+N(s) \cos \theta(s)\right) \theta_{1}(s) d s \\
& +\left.\beta \theta^{\prime} \theta_{1}\right|_{0} ^{L} .
\end{aligned}
$$

The integral in (2.8) must vanish for all $\theta_{1}$. Thus we get

$$
\beta \theta^{\prime \prime}+N \cos \theta=0, \quad \theta^{\prime}(0)=\theta^{\prime}(L)=0 .
$$


From (2.1), (2.2), (2.6), and (2.9), we see that the equilibrium equations are

$\theta^{\prime \prime}=-\frac{N}{\beta} \cos \theta, \quad N^{\prime}=-f(y), \quad y^{\prime}=\sin \theta$,

$\theta^{\prime}(0)=\theta^{\prime}(L)=0, \quad N(0)=0, \quad N(L)=v$,

where $f=-F^{\prime}$.

Recall that $F$ describes the van der Waals interaction between points on the sheet and the substrate. We describe briefly next the derivation of the specific form of $F$-see (Wilber et al., 2007b) for details. Because we assume that the substrate is formed by a second rigid graphene sheet, to define $F$ we start with a LennardJones 6-12 potential for the energy between two non-bonded carbon atoms. We multiply this potential by the atomic density of graphene, denoted $\sigma$, and integrate over the substrate. Multiplying the result by $\sigma$ again to get the energy per unit area between the sheet and the substrate, we arrive at

$F(\xi)=2 \pi \sigma^{2}\left(\frac{c_{12}}{10 \xi^{10}}-\frac{c_{6}}{4 \xi^{4}}\right), \quad f(\xi)=-F^{\prime}(\xi)=2 \pi \sigma^{2}\left(\frac{c_{12}}{\xi^{11}}-\frac{c_{6}}{\xi^{5}}\right)$,

where $c_{12}=3.859 \times 10^{-9} \mathrm{nN} \mathrm{nm}^{13}, c_{6}=2.43 \times 10^{-6} \mathrm{nN} \mathrm{nm}^{7}$, and $\sigma=38.177 \mathrm{~nm}^{-2}$ (Girifalco and Lad, 1956). The graphs of $F$ and $F^{\prime}$ are shown in Figs. 3 and 4. We note that our continuum approximation of the van der Waals interaction is not accurate near the substrate, where the effects of individual atoms at given positions on the substrate cause significant variations in the force. Note also that we model only the van der Waals interactions between atoms on the sheet and atoms on the substrate and ignore self-interactions within the sheet.

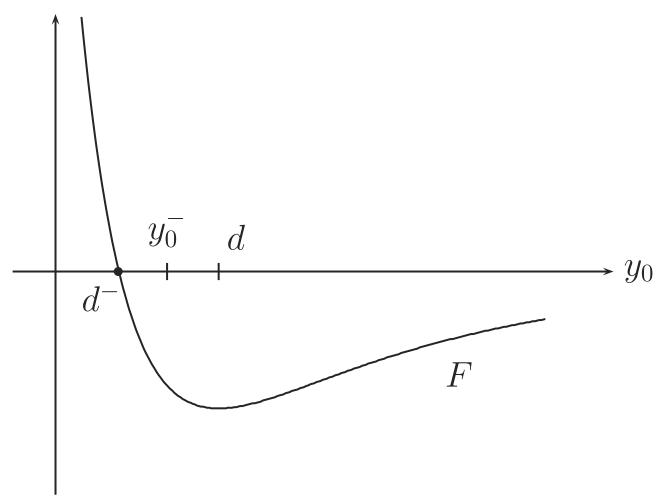

Fig. 3. Graph of $F$.

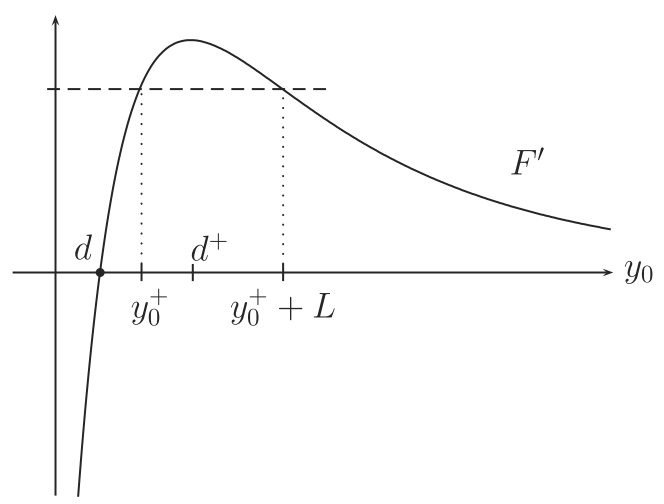

Fig. 4. Graph of $F^{\prime}$
Now we seek 'vertical solutions' to (2.10), i.e., solutions for which the sheet is straight and perpendicular to the substrate. For such solutions, $\theta(s)=\pi / 2$ for all $s \in[0, L]$. Substituting $\theta=\pi / 2$ into (2.10) yields

$N(s)=-\int_{0}^{s} f\left(y_{0}+\xi\right) d \xi, \quad y(s)=y_{0}+s$.

By $(2.12)_{1},(2.10 \mathrm{~b})_{3}$, and $(2.11)_{2}$, we see that a vertical solution exists if $y_{0}$ and $v$ satisfy

$v=-\int_{0}^{L} f\left(y_{0}+\xi\right) d \xi=F\left(y_{0}+L\right)-F\left(y_{0}\right)$,

which says merely that the applied load at the top of the sheet is balanced by the force from the interaction of the sheet with the substrate. Fig. 9 shows the set of solutions $\left(v, y_{0}\right)$ to (2.13) for a typical value of $L$. Note that for a given $L$, a vertical solution can be identified by the pair $\left(\pi / 2, y_{0}\right)$ describing its configuration or by the pair $\left(v, y_{0}\right)$ satisfying $(2.13)$.

\section{Stability and bifurcation}

Our goal now is to determine the stability of the vertical solutions identified at the end of the previous section. Exploiting our variational approach, we define a configuration $\left(\theta, y_{0}\right)$ as stable if it minimizes locally the total energy $E$ defined in (2.3). Furthermore, we assume that minimizers correspond to pairs $\left(\theta, y_{0}\right)$ at which the second variation of $E$ is positive definite. For a given length $L$, we shall find conditions on $y_{0}$ such that the vertical solution $\left(\pi / 2, y_{0}\right)$ is either stable or unstable.

\subsection{Computing the second variation}

We consider the energy of a configuration $\left(\pi / 2+\epsilon \delta \theta_{0}, y_{0}\right.$ $\left.+\epsilon \delta y_{0}\right)$, which corresponds to a vertical solution $\left(\pi / 2, y_{0}\right)$ subject to a small perturbation $\epsilon\left(\delta \theta_{0}, \delta y_{0}\right)$. Note that by (2.13), $y_{0}$ determines the applied load $v$ that appears in the following expressions. Using (2.3), we see that

$$
\begin{aligned}
\frac{\partial}{\partial \epsilon} E & {\left[\frac{\pi}{2}+\epsilon \delta \theta_{0}, y_{0}+\epsilon \delta y_{0}\right] } \\
= & \int_{0}^{L}\left[\beta \delta \theta_{0}^{\prime}\left(\epsilon \delta \theta_{0}^{\prime}\right)+F^{\prime}\left(y_{0}+\epsilon \delta y_{0}+\int_{0}^{s} \cos \left(\epsilon \delta \theta_{0}\right) d \xi\right)\right. \\
& \left.\times\left(\delta y_{0}-\int_{0}^{s} \delta \theta_{0} \sin \left(\epsilon \delta \theta_{0}\right) d \xi\right)\right] d s \\
& -v\left(\delta y_{0}-\int_{0}^{L} \delta \theta_{0} \sin \left(\epsilon \delta \theta_{0}\right) d s\right)
\end{aligned}
$$

and that

$$
\begin{aligned}
\frac{\partial^{2}}{\partial \epsilon^{2}} E & {\left[\frac{\pi}{2}+\epsilon \delta \theta_{0}, y_{0}+\epsilon \delta y_{0}\right] } \\
= & \int_{0}^{L}\left\{\beta\left(\delta \theta_{0}^{\prime}\right)^{2}+F^{\prime \prime}\left(y_{0}+\epsilon \delta y_{0}+\int_{0}^{s} \cos \left(\epsilon \delta \theta_{0}\right) d \xi\right)\right. \\
& \times\left(\delta y_{0}-\int_{0}^{s} \delta \theta_{0} \sin \left(\epsilon \delta \theta_{0}\right) d \xi\right)^{2} \\
& \left.+F^{\prime}\left(y_{0}+\epsilon \delta y_{0}+\int_{0}^{s} \cos \left(\epsilon \delta \theta_{0}\right) d \xi\right)\left(-\int_{0}^{s} \delta \theta_{0}^{2} \cos \left(\epsilon \delta \theta_{0}\right) d \xi\right)\right\} d s \\
& +v\left(\int_{0}^{L} \delta \theta_{0}^{2} \cos \left(\epsilon \delta \theta_{0}\right) d s\right) .
\end{aligned}
$$

If we let $\epsilon=0$, use (2.13), and note that

$\int_{0}^{L} F^{\prime \prime}\left(y_{0}+s\right) \delta y_{0}^{2} d s=\delta y_{0}^{2}\left[F^{\prime}\left(y_{0}+L\right)-F^{\prime}\left(y_{0}\right)\right]$

and that 


$$
\begin{aligned}
-\int_{0}^{L} F^{\prime}\left(y_{0}+s\right) \int_{0}^{s} \delta \theta_{0}^{2}(\xi) d \xi d s= & -F\left(y_{0}+L\right) \int_{0}^{L} \delta \theta_{0}(\xi) d \xi \\
& +\int_{0}^{L} F\left(y_{0}+s\right) \delta \theta_{0}^{2}(s) d s,
\end{aligned}
$$

we find that the second variation of $E$ at the vertical solution $\left(\pi / 2, y_{0}\right)$ is

$$
\begin{aligned}
G\left[\delta \theta_{0}, \delta y_{0}\right]:= & \int_{0}^{L}\left[\beta\left(\delta \theta_{0}^{\prime}(s)\right)^{2}+\left[F\left(y_{0}+s\right)-F\left(y_{0}\right)\right] \delta \theta_{0}^{2}(s)\right] d s \\
& +\delta y_{0}^{2}\left[F^{\prime}\left(y_{0}+L\right)-F^{\prime}\left(y_{0}\right)\right] .
\end{aligned}
$$

\subsection{Preliminary stability results}

We analyze (3.5) for fixed $L$ to determine the values of $y_{0}$ at which the corresponding vertical solution is stable. Our arguments are based on Figs. 3 and 4, which depict the graphs of $F$ and $F^{\prime}$.

First we show that there is a positive number $y_{0}^{+}$such that any vertical solution $\left(\pi / 2, y_{0}\right)$ with $y_{0}>y_{0}^{+}$is unstable. Let $d^{+}$be the point at which $F^{\prime}$ attains its maximum and let $d$ be the zero of $F^{\prime}$. See Fig. 4. Define $y_{0}^{+}$to be the unique point such that $F^{\prime}\left(y_{0}^{+}\right)=F^{\prime}\left(y_{0}^{+}+L\right)$. Note that $d<y_{0}^{+}<d^{+}$and that $y_{0}^{+}$depends on $L$. Suppose $y_{0}>y_{0}^{+}$. Then $F^{\prime}\left(y_{0}+L\right)<F^{\prime}\left(y_{0}\right)$. In the right-hand side of (3.5) we choose $\delta y_{0} \neq 0$ and $\delta \theta_{0}=0$. It follows that $G\left[\delta \theta_{0}, \delta y_{0}\right]=\frac{\delta y_{0}^{2}}{2}\left[F^{\prime}\left(y_{0}+L\right)-F^{\prime}\left(y_{0}\right)\right]<0$, and hence for any $y_{0}>y_{0}^{+}$, the corresponding vertical solution is unstable. The physical interpretation of these observations is that the vertical configuration of the sheet, if the sheet is sufficiently far from the substrate, is unstable with respect to small perturbations corresponding to translations perpendicular to the substrate.

We next show that there is a positive number $y_{0}^{-}$such that any vertical solution with $y_{0}<y_{0}^{-}$is unstable. Let $d^{-}$be the unique zero of $F$. From Fig. 3, we see that $d^{-}<d$. For $y_{0}>0$, define

$K\left(y_{0}, L\right):=\int_{0}^{L}\left[F\left(y_{0}+s\right)-F\left(y_{0}\right)\right] d s$.

If $y_{0}<d$, then

$\frac{\partial K\left(y_{0}, L\right)}{\partial y_{0}}=F\left(y_{0}+L\right)-F\left(y_{0}\right)-L F^{\prime}\left(y_{0}\right)>0$,

where the last inequality follows because $F$ is concave up on $(0, d)$. Also, $K\left(d^{-}, L\right)<0$ and $K(d, L)>0$. Hence there is a unique point $y_{0}^{-}$ between $d^{-}$and $d$ such that $K\left(y_{0}^{-}, L\right)=0$ and $K\left(y_{0}, L\right)<0$ if $0<y_{0}<y_{0}^{-}$. If we choose $\delta y_{0}=0$ and $\delta \theta_{0}=k$, where $k \neq 0$ is a constant, then $G\left[\delta \theta_{0}, \delta y_{0}\right]=k^{2} K\left(y_{0}, L\right)<0$, confirming that for any $0<y_{0}<y_{0}^{-}$, the corresponding vertical solution is unstable. Physically, if the bottom edge of the sheet is sufficiently close to the substrate, then the vertical configuration is unstable with respect to small perturbations corresponding to tilting the sheet.

Next, from Fig. 4 , we see that $\frac{\delta y_{0}^{2}}{2}\left[F^{\prime}\left(y_{0}+L\right)-F^{\prime}\left(y_{0}\right)\right] \geqslant 0$ for any separation $y_{0}$ with $d \leqslant y_{0} \leqslant y_{0}^{+}$. From Fig. 3 we see that $F\left(y_{0}+s\right)-F\left(y_{0}\right)>0$ for $d \leqslant y_{0}$ and $s>0$. Therefore, because all three terms in $G$ are non-negative and at least one is positive for any choice of $\delta \theta$ and $\delta y$ not both 0 , we conclude that vertical solutions corresponding to $y_{0}$ with $d \leqslant y_{0} \leqslant y_{0}^{+}$are stable.

\subsection{Bifurcation}

Summarizing the results of the previous subsection, we know that for a given $L$ there are numbers $y_{0}^{-}$and $y_{0}^{+}$with $y_{0}^{-}<d<y_{0}^{+}$ such that a vertical solution $\left(\pi / 2, y_{0}\right)$ with either $y_{0}<y_{0}^{-}$or $y_{0}>y_{0}^{+}$is unstable and a vertical solution $\left(\pi / 2, y_{0}\right)$ with $d \leqslant y_{0} \leqslant y_{0}^{+}$is stable. Next we determine the stability of a vertical solution with $y_{0}$ between $y_{0}^{-}$and $d$. We use basic ideas from bifur- cation theory to demonstrate that, for a given $L$, there is a unique bifurcation point at a vertical solution $\left(\pi / 2, y_{0}^{*}\right)$ where $y_{0}^{*}$ satisfies $y_{0}^{-}<y_{0}^{*}<d$. From this we deduce the stability of vertical solutions $\left(\pi / 2, y_{0}\right)$ with $y_{0}$ between $y_{0}^{-}$and $d$. Also we identify additional bifurcation points outside the interval $\left(y_{0}^{-}, d\right)$.

We proceed by taking as our trivial branch the family of vertical solutions parametrized by $y_{0}$ for $y_{0}>0$. We seek parameter values at which the linearized equations have nontrivial solutions. We assume that these values are bifurcation points. To linearize (2.10) around a typical solution (2.12) on the trivial branch, we define

$N_{0}(s):=-\int_{0}^{s} f\left(y_{0}+\xi\right) d \xi$

substitute

$N=N_{0}+\epsilon N_{1}, \quad y=y_{0}+s+\epsilon y_{1}, \quad \theta=\frac{\pi}{2}+\epsilon \theta_{1}$

into (2.10), take a derivative with respect to $\epsilon$, and set $\epsilon=0$ to obtain

$\theta_{1}^{\prime \prime}=\frac{N_{0}}{\beta} \theta_{1}, \quad N_{1}^{\prime}=-f^{\prime}\left(y_{0}+s\right) y_{1}, \quad y_{1}^{\prime}=0$.

Likewise we linearize the boundary conditions to obtain

$N_{1}(0)=N_{1}(L)=0, \quad \theta_{1}^{\prime}(0)=\theta_{1}^{\prime}(L)=0$.

To discover where problem (3.10) has non-trivial solutions, we first note that $y_{1}$ is constant by $(3.10)_{3}$. Next, integrating $(3.10)_{2}$ from 0 to $s$ and using (3.11) yields

$N_{1}(s)=-\int_{0}^{s} f^{\prime}\left(y_{0}+\xi\right) y_{1} d \xi$.

We then apply $(3.11)_{1}$ again to obtain

$0=-\int_{0}^{L} f^{\prime}\left(y_{0}+\xi\right) y_{1} d \xi=y_{1}\left(F^{\prime}\left(y_{0}+L\right)-F^{\prime}\left(y_{0}\right)\right)$.

Eq. (3.13) implies that either $y_{0}=y_{0}^{+}$(see Fig. 4) or $y_{1}=0$. If $y_{0}=y_{0}^{+}$, then choosing $y_{1} \neq 0$ yields a non-trivial solution to (3.10) and (3.11). Hence $y_{0}^{+}$is a bifurcation point. We know from the previous subsection that the stability of the trivial branch changes at $y_{0}^{+}$.

On the other hand, if $y_{1}=0$, then (3.12) implies $N_{1}(s)=0$ for all $s \in[0, L]$. Hence for $y_{0} \neq y_{0}^{+},(3.10)$ and (3.11) has a non-trivial solution if and only if $(3.10)_{1}$ and $(3.11)_{2}$ has a non-trivial solution. Eq. $(3.10)_{1}$ is a linear non-constant coefficient equation in which the parameter $y_{0}$ appears nonlinearly, and with $(3.11)_{2}$ it is in the form of a classical, self-adjoint two-point boundary-value problem. We organize our results about $(3.10)_{1},(3.11)_{2}$ by considering the set of eigencurves in the $L y_{0}$ - plane, i.e., curves defined by combinations of $L$ and $y_{0}$ at which (3.10) $)_{1}$ and (3.11) 2 has a non-trivial solution. We use the shooting method as well as the numerical bifurcation software AUTO (Doedel et al., 2006) to locate these eigencurves numerically. See Fig. 5. We divide the first quadrant of the $L y_{0}$ - plane into two regions $R_{1}$ and $R_{2}$ above and below the line $y_{0}=d^{-}$. In $R_{1}$, there is a unique eigencurve, which can be described as the graph of a function $L \mapsto c_{0}(L)$. The function $c_{0}$ is strictly decreasing, $c_{0}(L) \rightarrow d$ as $L \rightarrow 0^{+}$, and $c_{0}(L)$ converges to a constant $D$ as $L \rightarrow \infty$, where $D>d^{-}$. The proofs of these observations are discussed in Appendix A.

Some numerical values of the bifurcation points are presented in Table 1. The numbers in Table 1 , as well as Fig. 5(b), indicate that for each $L, y_{0}^{*}=c_{0}(L)$ is the unique bifurcation point between $y_{0}^{-}$ and $d$. Adopting a standard point of view in the study of structural stability, we interpret $y_{0}^{*}$ as the value at which solutions on the trivial branch lose stability. Hence, we assume that the stable region $d \leqslant y_{0} \leqslant y_{0}^{+}$identified in Section 3.2 extends to $y_{0}^{*}<y_{0} \leqslant y_{0}^{+}$ and that the unstable region $y_{0}<y_{0}^{-}$identified in the previous section extends to $y_{0}<y_{0}^{*}$. 


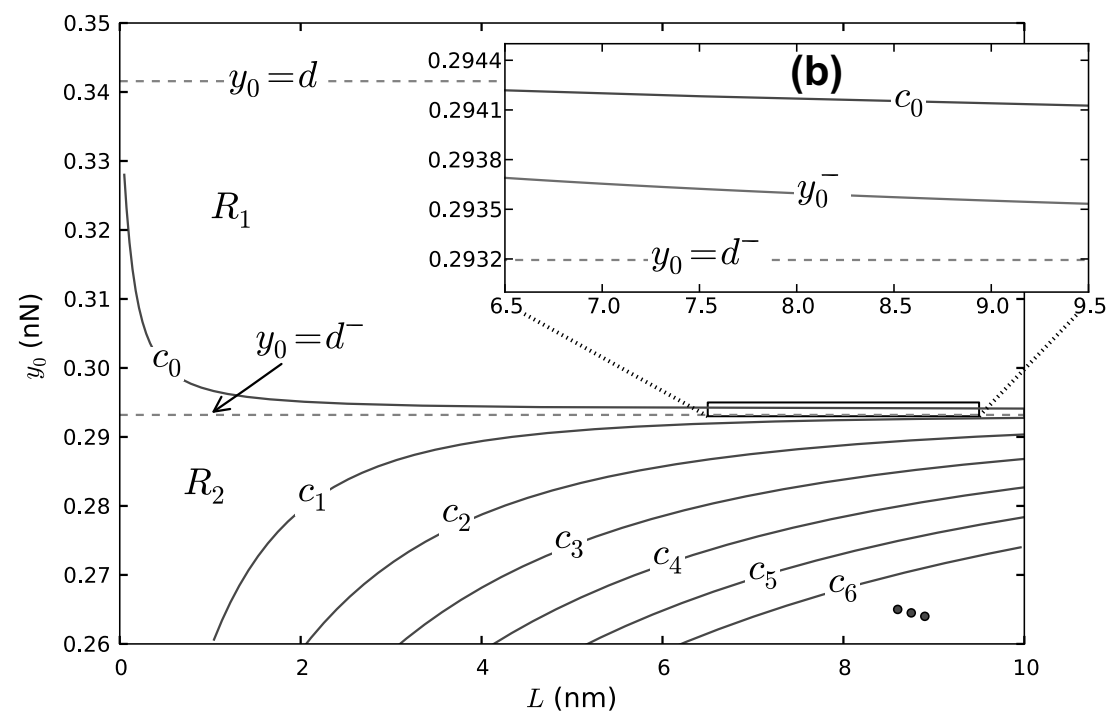

(a)

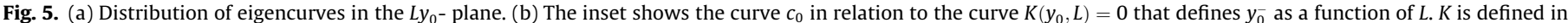
(3.6).

Table 1

Bifurcation points for various lengths $L$. We let $v^{*}$ denote the load corresponding to $y_{0}^{*}$. See Fig. 9. Numbers in the first 4 columns have units of nm. Numbers in the final column have units of $\mathrm{nN} / \mathrm{nm}$.

\begin{tabular}{rllll}
\hline \multicolumn{1}{c}{$L$} & $y_{0}^{-}$ & $y_{0}^{*}=c_{0}(L)$ & $d$ & $v^{*}$ \\
\hline 5 & 0.293841 & 0.29429120361 & 0.341569 & .016433694 \\
25 & 0.293322 & 0.29415195334 & 0.341569 & .014405597 \\
150 & 0.293215 & 0.29415195200 & 0.341569 & .014405591 \\
\hline
\end{tabular}

Additional bifurcation points lie in $R_{2}$ on a countable number of eigencurves $L \mapsto c_{k}(L)$ for $k=1,2, \ldots$. See Fig. 5 . For each $k$, the function $c_{k}$ is strictly increasing and $c_{k}(L) \rightarrow d^{-}$as $L \rightarrow \infty$. Also, for any fixed $L, c_{k}(L)>c_{k+1}(L)$ for $k=1,2, \ldots$. An eigenfunction corresponding to a point on the graph of $c_{k}$ has exactly $k$ zeros. See Appendix A for comments on the proof of these observations.

\subsection{Buckled configurations}

Next we compute the buckled configurations corresponding to bifurcation points on different eigencurves. We first introduce some useful terminology. Using (2.13), for any bifurcation point $y_{0}$ we can find the corresponding applied load $v$. If the load corresponding to $y_{0}$ is positive, then this load is pulling the sheet away from the substrate. In this case, we shall refer to $y_{0}$ as a "pulling bifurcation point" and the associated non-trivial solutions as "pulling solutions". Likewise, we use "pushing bifurcation point" and "pushing solutions" if the load corresponding to $y_{0}$ is negative.

Whether a given bifurcation point is pulling or pushing is determined by whether the right-hand side of $(2.13)$ is positive or negative. The level curve $F\left(y_{0}+L\right)-F\left(y_{0}\right)=0$ in the $L y_{0}$ - plane lies between the curve $c_{0}$ and the line $y_{0}=d^{-}$in Fig. 5 with $F\left(y_{0}+L\right)-F\left(y_{0}\right)>0$ above this level set and $F\left(y_{0}+L\right)-F\left(y_{0}\right)<0$ below this level set. (This level set is not depicted in Fig. 5(b) because it would lie essentially on top of the line $y_{0}=d^{-}$.) It follows that bifurcation points on $c_{0}$ are pulling (see also the last column in Table 1 ) and that bifurcation points on $c_{k}$ for $k=1,2, \ldots$ are pushing.

The pulling solutions corresponding to pulling bifurcation points on $c_{0}$ for several different lengths are depicted in Figs. 6

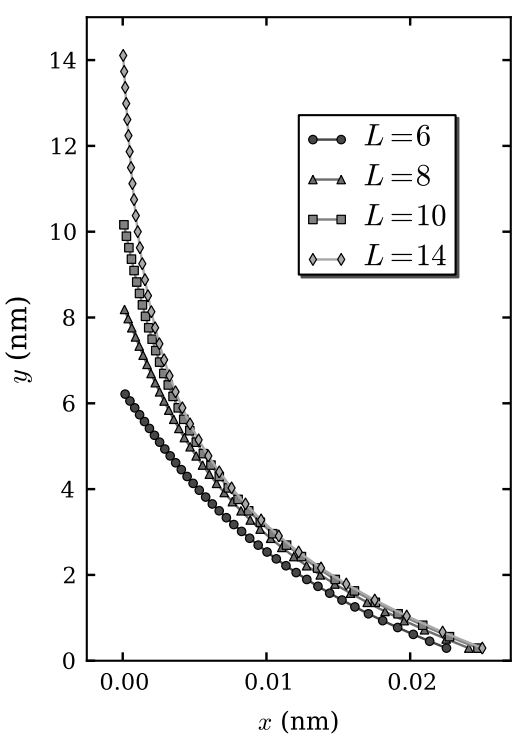

Fig. 6. Buckled configurations corresponding to the pulling bifurcation points for sheets of lengths $L=6,8,10$, and 14 .

and 7. Sheets of smaller lengths tilt in relation to the substrate. For longer sheets, only the bottom $25 \mathrm{~nm}$ or so deflect or kick out to one side, while the rest of the sheet remains vertical. All additional bifurcation points for $(3.10)_{1}$ and $(3.11)_{2}$ are pushing bifurcations. Hence in particular $y_{0}^{*}$ and $y_{0}^{+}$are the only pulling bifurcation points. (We do not show plots of 'buckled' configurations corresponding to $y_{0}^{+}$-such configurations are straight lines. See (3.9) and the paragraph containing (3.12).).

In Fig. 8 we show buckled configurations for the first several pushing bifurcation points for $L=6$ and $L=60$. Additional pushing bifurcation points have corresponding buckled solutions that increase in mode number, i.e., increase in the number of half sine waves in the buckled configuration. This is analogous to what one observes in the classical Euler buckling of a beam with, say, hinged boundary conditions. Note, however, that for the pushing 


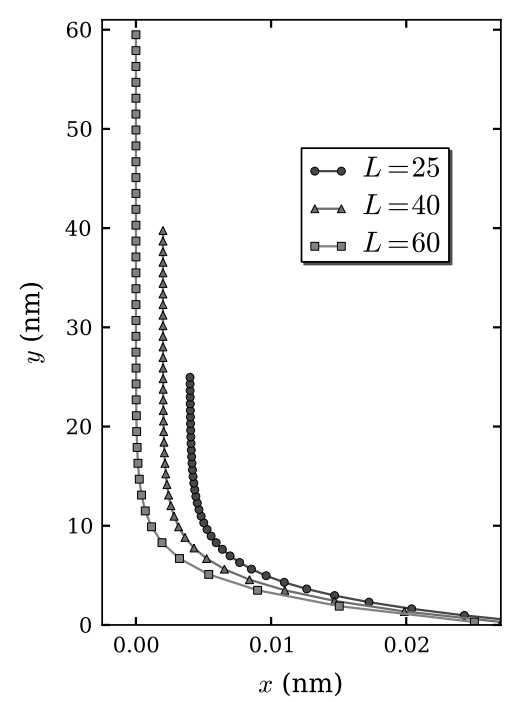

Fig. 7. Buckled configurations corresponding to the pulling bifurcation points for sheets of lengths $L=25,40$, and 60 .

bifurcations the bottom of the sheet does not sit directly below the top of the sheet but rather is shifted horizontally.

Remark. The expression for the van der Waals interaction force used in Strus and Raman (2009) is derived for a carbon nanotube, rather than a sheet, interacting with a substrate and hence has the same form as our expression $(2.11)_{2}$ but with different constants. Our analysis and computations would yield the same qualitative results if the parameters in our problem were changed to the values appropriate for a carbon nanotube used in Strus and Raman (2009). The authors in Strus and Raman (2009) study a 'pinned' case that describes how the nanotube transitions between different configurations when the tip sticks to the surface rather than sliding along the surface as in their perfect slip case. They model sticking by changing the boundary condition from $(2.10 \mathrm{~b})_{2}$ to $N(0)=K x(0)$, where $K$ is a constant (cf. (4) in Strus and Raman (2009)). Hence to model sticking they explicitly assume that the tip has stuck by introducing a force in addition to the van der
Waals interaction. If one retains $(2.10 \mathrm{~b})_{2}$, then the model used here and in Strus and Raman (2009) predicts deformations that look like sticking, which we call the pushing solutions. Compare Fig. 8 above to Fig. 1(b) in Strus and Raman (2009). It is interesting to note, however, that the pushing solutions occur only after the pulling, or sliding, solution at bifurcation points on an unstable section of the trivial branch.

\subsection{Validation}

Here we note available experimental results from the literature that validate the predictions of our model. In Strus and Raman (2009), the authors perform experiments in which atomic force microscopes with carbon nanotube tips are pushed into and then retracted from rigid surfaces of several different materials. Based on force-distance data, these authors deduce that the nanotube at certain distances assumes a configuration corresponding to our pulling solution. Likewise, the nanotube at certain distances assumes a configuration corresponding to our first pushing bifurcation. See Fig. 3 in Strus and Raman (2009). Similar experimental results are presented (Yap et al., 2007). In Evans and Lauga (2009), the authors describe a macroscale experiment with charged adhesive tape and a flat surface. As the tape is moved toward the surface, a transition occurs similar to our pulling bifurcation-see Fig. 1 in Evans and Lauga (2009)-although we note that the boundary condition at the top of the tape does not appear to match the condition we use at the top of the sheet. We are unaware of any experiments with graphene sheets that validate our theoretical results.

\subsection{Summary of stability and bifurcation results}

The results of this section are summarized in Fig. 9, which shows for a typical value of $L$ the pairs $\left(v, y_{0}\right)$ at which a vertical solution exists (i.e., pairs satisfying (2.13)). The dashed lines in Fig. 9 indicate points $\left(v, y_{0}\right)$ where the corresponding vertical solution $\left(\pi / 2, y_{0}\right)$ is unstable, while the solid line indicates points where the corresponding vertical solution is stable. Vertical solutions lose stability as the parameter $y_{0}$ is decreased from above $y_{0}^{*}$ to below $y_{0}^{*}$. Likewise, vertical solutions lose stability as $y_{0}$ is in-

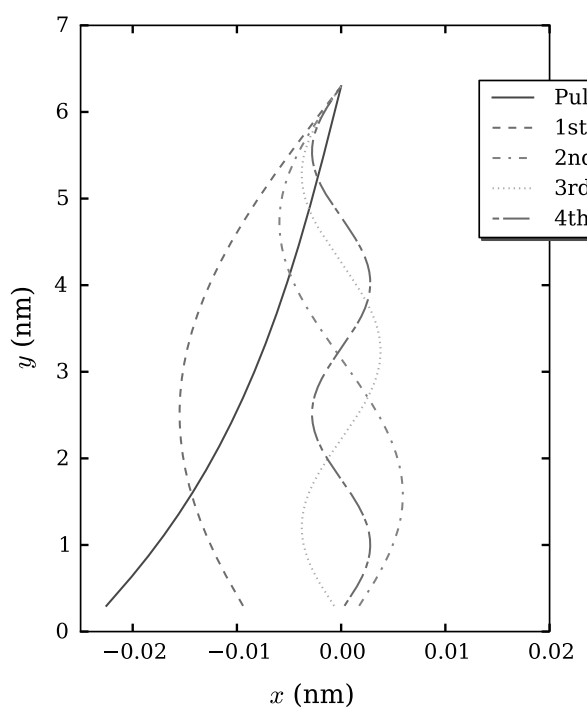

(a)

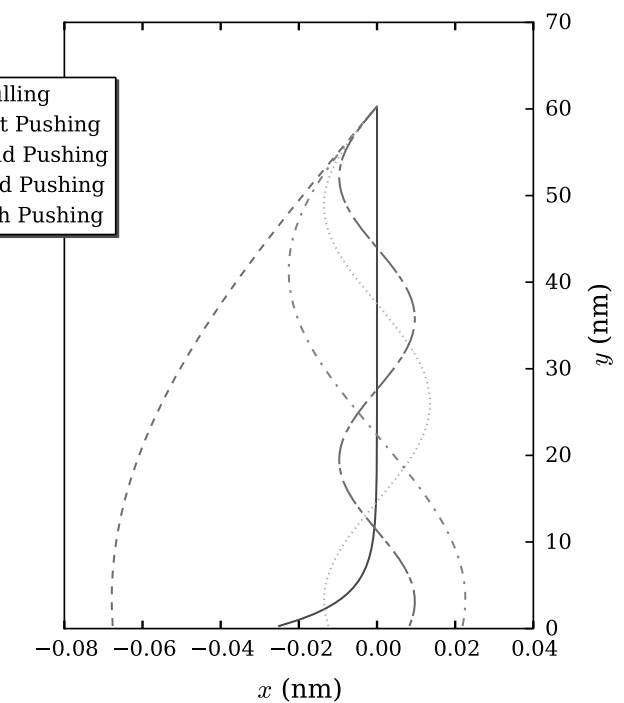

(b)

Fig. 8. The first five buckled configurations for (a) $L=6$ and (b) $L=60$. 


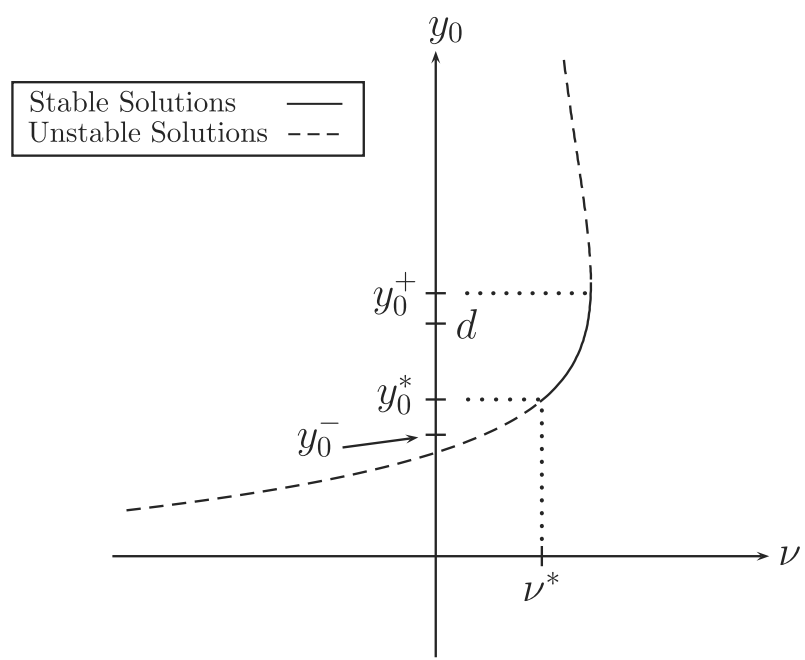

Fig. 9. Stability of vertical solutions for typical length $L$. The curve is the set of pairs $\left(v, y_{0}\right)$ that satisfy (2.13). For each point on the curve, dashed versus solid indicates the stability of the corresponding vertical solution $\left(\pi / 2, y_{0}\right)$.

creased from below $y_{0}^{+}$to above $y_{0}^{+}$. We identified additional bifurcation points, all of which lie on the part of the curve in Fig. 9 to the left of the $y_{0}$-axis. Recall that in Section 1 we described an experiment that places a physical interpretation on these results. Note that any point on the stable section of the curve in Fig. 9 can serve as the starting point for the experiment described in Section 1.

\section{Bifurcations for long sheets}

In this section, we study our problem for long sheets. Because the van der Waals force decays quickly to zero, if $L$ is large, only a relatively small part of the bottom of the sheet interacts with the substrate. We introduce a small parameter $\epsilon$ to treat the interacting bottom part of the sheet as a boundary layer. We show that if the applied load is $O\left(\epsilon^{2}\right)$, the pushing bifurcations appear in the outer problem as solutions to a classical Euler buckling problem. However, if the applied load is $O(1)$, then only the two pulling bifurcations arise, and these occur in the inner problem.

To rescale (2.10), we use

$\hat{s}=\frac{s}{L}, \quad \hat{\theta}=\theta, \quad \hat{v}=d v, \quad \widehat{N}=\frac{N}{\bar{v}}$,

$\hat{y}=\frac{y}{L}, \quad \hat{\beta}=\frac{\beta}{\bar{v} d^{2}}, \quad \hat{f}(\xi)=\frac{f(d \xi) d}{\bar{v}}, \quad \hat{v}=\frac{v}{\bar{v}}$.

Recall that $d=.3416 \mathrm{~nm}$ is the unique zero of $f$. Also, we take as our characteristic load $\bar{v}=-F(d)=.2452 \mathrm{nN} \mathrm{nm}^{-1}$. We apply these rescalings to get the non-dimensional system

$\epsilon \hat{v}^{\prime}=-\frac{\widehat{N}}{\hat{\beta}} \cos (\hat{\theta}), \quad \epsilon \hat{\theta}^{\prime}=\hat{v}$,

$\epsilon \widehat{N}^{\prime}=-\hat{f}(\hat{y} / \epsilon), \quad \hat{y}^{\prime}=\sin (\hat{\theta})$,

$\widehat{N}(0)=0, \quad \widehat{N}(1)=\hat{v}, \quad \hat{v}(0)=\hat{v}(1)=0$,

where $\epsilon=d / L$ is our small parameter.

In the usual way, to study the outer problem we introduce expansions $\hat{v}=\hat{v}_{0}+\epsilon \hat{v}_{1}+\epsilon^{2} \hat{v}_{2}+\cdots$, etc. In particular we expand the applied load as $\hat{v}=\hat{v}_{0}+\epsilon \hat{v}_{1}+\epsilon^{2} \hat{v}_{2}+\cdots$. Substituting these expansions into (4.3)-(4.5) and enforcing only the boundary conditions at the top of the sheet, we find at $O(1)$ that $\hat{v}_{0}=0, \quad-\frac{\widehat{N}_{0}}{\hat{\beta}} \cos \left(\hat{\theta}_{0}\right)=0, \quad \hat{y}_{0}^{\prime}=\sin \left(\hat{\theta}_{0}\right)$,

$\widehat{N}_{0}(1)=\hat{v}_{0}, \quad \hat{v}_{0}(1)=0$,

and at $O(\epsilon)$ that

$\hat{v}_{0}^{\prime}=-\frac{1}{\hat{\beta}}\left(\widehat{N}_{1} \cos \left(\hat{\theta}_{0}\right)-\widehat{N}_{0} \sin \left(\hat{\theta}_{0}\right) \hat{\theta}_{1}\right), \quad \hat{\theta}_{0}^{\prime}=\hat{v}_{1}$,

$\widehat{N}_{0}^{\prime}=0, \quad \hat{y}_{1}^{\prime}=\cos \left(\hat{\theta}_{0}\right) \hat{\theta}_{1}$,

$\widehat{N}_{1}(1)=\hat{v}_{1}, \quad \hat{v}_{1}(1)=0$.

Suppose the applied load is $O(1)$, so that $\hat{v}_{0} \neq 0$ and $\hat{v}_{j}=0$ for $j \geqslant 1$. Eq. (4.6) $)_{1}$ tells us that $\hat{v}_{0} \equiv 0$. Also, $(4.9)_{1}$ and $(4.7)_{1}$ imply that $\widehat{N}_{0} \equiv \hat{v}_{0}$. We choose the straight configuration $\hat{\theta}_{0} \equiv \pi / 2$ as the base state, so that $(4.6)_{2}$ is satisfied. Then $(4.8)_{1}$ implies that $\hat{\theta}_{1} \equiv 0$. Eq. $(4.9)_{2}$ implies that $\hat{y}_{1}$ is constant, and matching shows that this constant is 0 . Hence if the applied load is $O(1)$, the outer problem yields no bifurcation points.

On the other hand, suppose the applied load is smaller than $O(1)$. Then $\hat{v}_{0}=0$ and hence $(4.7)_{1}$ with $(4.9)_{1}$ imply that $\widehat{N}_{0} \equiv 0$. In this case, $(4.8)_{1}$ gives no information on $\hat{\theta}_{1}$. If the applied load is of $O\left(\epsilon^{2}\right)$, so that $\hat{v}_{0}=\hat{v}_{1}=0$ and $\hat{v}_{2} \neq 0$, we discover by computing the $O\left(\epsilon^{2}\right)$ problem that $\hat{\theta}_{0}$ satisfies

$\hat{\theta}_{0}^{\prime \prime}=-\hat{\beta}^{-1} \hat{v}_{2} \cos \hat{\theta}_{0}, \quad \hat{\theta}_{0}^{\prime}(L)=0$.

Again we take $\hat{\theta}_{0}=\pi / 2$ as our base state. By computing the $O\left(\epsilon^{3}\right)$ problem, we further discover that

$\hat{\theta}_{1}^{\prime \prime}=-\hat{\beta}^{-1} \hat{v}_{2} \hat{\theta}_{1}, \quad \hat{\theta}_{1}^{\prime}(L)=0$.

Upon supplementing each of (4.11) and (4.12) with an additional boundary condition obtained by matching, we see that there are a countable number of bifurcation points $\hat{v}_{2}>0$. These correspond to the pushing bifurcations found in the previous section. Eq. (4.11) is a version of the classical Euler buckling problem.

To write down the inner problem, we introduce the additional rescalings

$\eta=\hat{s} / \epsilon, \quad y=\hat{y} / \epsilon$.

From (4.3)-(4.5), we get

$v^{\prime}=-\frac{N}{\hat{\beta}} \cos (\theta), \quad N^{\prime}=-\hat{f}(y)$,

$y^{\prime}=\sin (\theta), \quad \theta^{\prime}=v$,

$N(0)=0, \quad N(1 / \epsilon)=\hat{v}, \quad v(0)=0, \quad v(1 / \epsilon)=0$.

Introducing expansions for the inner variables yields the $O(1)$ inner problem

$v_{0}^{\prime}=-\frac{N_{0}}{\hat{\beta}} \cos \left(\theta_{0}\right), \quad N_{0}^{\prime}=-\hat{f}\left(y_{0}\right)$,

$y_{0}^{\prime}=\sin \left(\theta_{0}\right), \quad \theta_{0}^{\prime}=v_{0}$,

$N_{0}(0)=0, \quad v_{0}(0)=0$.

Note that this is the original nonlinear boundary-value problem (2.10) without the boundary conditions at the top of the sheet. As we did for (2.10), here we can identify a base-state solution, namely, $v_{0} \equiv 0, y_{0}(\eta)=Y_{0}+\eta, N_{0}(\eta)=-\int_{0}^{\eta} \hat{f}\left(Y_{0}+\xi\right) d \xi$, and $\theta_{0} \equiv \pi / 2$. We assume the applied load $\hat{v}$ is $O(1)$. Matching in the usual way implies that $N_{0}(\eta \rightarrow \infty)=-\int_{0}^{\infty} \hat{f}\left(Y_{0}+\xi\right) d \xi=\hat{v}_{0}$, which determines the constant $Y_{0}$.

At $O(\epsilon)$, we have

$v_{1}^{\prime}=\frac{N_{0}}{\beta} \theta_{1}, \quad N_{1} \prime=-\hat{f}^{\prime}\left(Y_{0}+\eta\right) y_{1}$,

$y_{1}^{\prime}=0, \quad \theta_{1}^{\prime}=v_{1}$,

$N_{1}(0)=0, \quad v_{1}(0)=0$. 
Values of $\hat{v}_{0}$ (which appears implicitly in (4.20)-(4.22) through the constant $Y_{0}$ ) at which the $O(\epsilon)$ problem has a non-trivial solution correspond to bifurcation points that arise from the inner problem. By $(4.21)_{1}, y_{1}$ is a constant. Integrating $(4.20)_{2}$ and using $(4.22)_{1}$ yield

$N_{1}(\eta)=-\int_{0}^{\eta} \hat{f}^{\prime}\left(Y_{0}+\xi\right) y_{1} d \xi$

Then by matching we obtain

$0=N_{1}(\eta \rightarrow \infty)=-\int_{0}^{\infty} \hat{f}^{\prime}\left(Y_{0}+\xi\right) y_{1} d \xi=y_{1} \hat{f}\left(Y_{0}\right)$

from which it follows that either $y_{1}=0$ or $Y_{0}=1$. (For the last equality in (4.24), recall $(4.2)_{3}$ and recall that $f(\xi) \rightarrow 0$ as $\left.\xi \rightarrow \infty\right)$. If $Y_{0}=1$, then $\hat{f}\left(Y_{0}\right)=0$ and $y_{1}$ is arbitrary, and hence the $O(\epsilon)$ problem has a non-trivial solution. We conclude that $Y_{0}=1$ is a bifurcation point. If $y_{1}=0$, then $N_{1} \equiv 0$ and hence for a given value of $Y_{0}$, (4.20)-(4.22) has a non-trivial solution if and only if $(4.20)_{1},(4.21)_{2}$, and $(4.22)_{3,4}$ has a non-trivial solution.

We write $(4.20)_{1},(4.21)_{2}$, and $(4.22)_{2}$ as the second-order problem

$\theta_{1}^{\prime \prime}-\frac{N_{0}}{\hat{\beta}} \theta_{1}=0, \quad \theta_{1}^{\prime}(0)=0$,

in which

$N_{0}(\eta)=-\int_{0}^{\eta} \hat{f}\left(Y_{0}+\xi\right) d \xi=\widehat{F}\left(Y_{0}+\eta\right)-\widehat{F}\left(Y_{0}\right), \quad N_{0}(\eta \rightarrow \infty)=\hat{v}$

where $\widehat{F}(y)=\bar{v}^{-1} F(d y)$. We use matching to derive for (4.25) the additional conditions

$\theta_{1}(\eta \rightarrow \infty)=0, \quad \theta_{1}^{\prime}(\eta \rightarrow \infty)=0$.

Next we show that there is a unique value of $Y_{0}$ at which (4.25), (4.27) has nontrivial solutions. For this argument, the reader may wish to consult the earlier Figs. 3 and 4, which depict the graphs of $F$ and $f$. These graphs are qualitatively the same as those of $\widehat{F}$ and $\hat{f}$. Note that in the current scalings, $Y_{0}=1$ and $Y_{0}=d^{-} / d$ correspond to $y_{0}=d$ and $y_{0}=d^{-}$in Figs. 3 and 4 .

First we show that there are no values of $Y_{0}$ in $[1, \infty)$ at which (4.25), (4.27) has nontrivial solutions. Let $\theta_{1}$ be a solution to (4.25). Multiplying both sides of $(4.25)_{1}$ by $2 \theta_{1}^{\prime}$ yields $2 \theta_{1}^{\prime} \theta_{1}^{\prime \prime}-2 \hat{\beta}^{-1} N_{0} \theta_{1} \theta_{1}^{\prime}=0$, which implies that

$\left(\left(\theta_{1}^{\prime}\right)^{2}\right)^{\prime}-\left(\frac{N_{0}}{\hat{\beta}} \theta_{1}^{2}\right)^{\prime}=-\frac{N_{0}^{\prime}}{\hat{\beta}} \theta_{1}^{2}=\frac{1}{\hat{\beta}} \hat{f}\left(Y_{0}+\eta\right) \theta_{1}^{2}$.

We then integrate both sides of (4.28) from 0 to $\infty$ and use $(4.25)_{2}$, $(4.26)_{1}$, and (4.27) to discover

$0=\int_{0}^{\infty} \frac{\hat{f}\left(Y_{0}+\xi\right)}{\hat{\beta}} \theta_{1}^{2}(\xi) d \xi$

which implies that $\theta_{1} \equiv 0$ since $\hat{f}\left(Y_{0}+\xi\right)<0$ for all $\xi>0$ if $Y_{0} \geqslant 1$. Therefore, for $Y_{0} \geqslant 1$, the function $\theta_{1} \equiv 0$ is the only solution of (4.25), (4.27).

Likewise, there are no values of $Y_{0}$ in $\left(0, d^{-} / d\right]$ at which (4.25), (4.27) has nontrivial solutions. In this case, $N_{0}(\eta)<0$ for all $\eta>0$, and if $\xi>1-Y_{0}$, then $\hat{f}\left(Y_{0}+\xi\right)<0$. Now we integrate both sides of Eq. (4.28) from $1-Y_{0}$ to $\infty$ and use $(4.25)_{2}$ and (4.26) 1 to discover

$$
\begin{aligned}
& -\left(\theta_{1}^{\prime}\left(1-Y_{0}\right)\right)^{2}+\frac{N_{0}\left(1-Y_{0}\right)}{\hat{\beta}} \theta_{1}^{2}\left(1-Y_{0}\right) \\
& =-\int_{1-Y_{0}}^{\infty} \frac{\hat{f}\left(Y_{0}+\xi\right)}{\hat{\beta}} \theta_{1}^{2}(\xi) d \xi .
\end{aligned}
$$

Note that the left-hand side of (4.30) is non-positive and the right-hand side is non-negative. Hence both sides must be zero, which is possible only if $\theta_{1} \equiv 0$. Thus, for $Y_{0} \in\left(0, d^{-} / d\right]$, there are no bifurcation points.

Lastly we consider $Y_{0} \in\left(d^{-} / d, 1\right)$. Here we note merely that by a shooting method one discovers numerically a unique value $Y_{0}^{*}$ between $d^{-} / d$ and 1 at which (4.25) and (4.27) has nontrivial solutions. Further details are provided in Appendix A. Because we have shown that no bifurcation points exist outside of $\left(d^{-} / d, 1\right)$, we conclude that $Y_{0}^{*}$ is the unique bifurcation point for (4.25) and (4.27). The point $Y_{0}^{*} \in\left(d^{-} / d, 1\right)$, hence the applied load $\hat{v}^{*}=-\widehat{F}\left(Y_{0}^{*}\right)$ is positive, so that $Y_{0}^{*}$ is a pulling bifurcation point. In fact $Y_{0}^{*}$ equals $\lim _{L \rightarrow \infty} c_{0}(L) / d$, where $c_{0}$ is described in Fig. 5. I.e., $Y_{0}^{*}$ is the limiting value as $L \rightarrow \infty$ of the unique pulling bifurcation point that exists for any finite $L$. (The factor $d^{-1}$ appears in the limit because of the rescaling used for the inner problem.).

\subsection{Summary of bifurcation results for large $L$}

The boundary-layer approach of this section reveals that for large $L$ the pulling and pushing bifurcations arise by different mechanisms. The two pulling bifurcations arise in the inner problem if the applied load is leading order. These bifurcations are novel and are tied to the van der Waals interaction between the sheet and the substrate. The countable number of pushing bifurcations arise in the outer problem if the applied load is $O\left(\epsilon^{2}\right)$, in which case the outer problem is a version of the classical Euler buckling problem.

\section{Acknowledgments}

This research was supported by the National Science Foundation through the grants DMS-0354022, DMS-0407361, and DMS-1009849. The authors thank J. Gallagher and Y. Milman, who participated in an REU project in which the problem studied in this paper was formulated.

\section{Appendix A. The properties of the eigencurve $c_{0}$}

In Section 3.3, we describe the properties of the curve $c_{0}$, justifying our claims by referring to Fig. 5 , in which the curve is constructed numerically. Here we comment on the proofs of these claims. The problem $(3.10)_{1},(3.11)_{2}$ can be studied by introducing the change of variables

$\theta=r \sin w, \quad \theta^{\prime}=r \cos w$.

(We are using ideas from the proofs of comparison theorems for ordinary differential equations. See, for example, Chapter 8 in Coddington and Levinson (1955).) One checks that $r$ and $w$ satisfy the system

$w^{\prime}=\cos ^{2} w+g \sin ^{2} w, \quad r^{\prime}=r(1-g) \sin w \cos w$,

where

$g\left(s, y_{0}\right)=-\beta^{-1} N_{0}(s)=\beta^{-1} \int_{0}^{s} f\left(y_{0}+\xi\right) d \xi$.

Solutions to (3.10) $)_{1}$ and $(3.11)_{2}$ correspond to solutions $w$ to the initial value problem $(5.2)_{1}, w(0)=\pi / 2$ for which there exists an $L>0$ with $w(L)=(2 k+1) \pi / 2$ for some integer $k$.

To study this latter problem, we note the basic properties of the vector field for $(5.2)_{1}$. In Fig. 10(a), the curve $\mathcal{H}_{1}$ is a subset of the horizontal isocline for the vector field. So on $\mathcal{H}_{1}$ the tangent vectors are horizontal. In the region outside this curve, the tangent vectors 


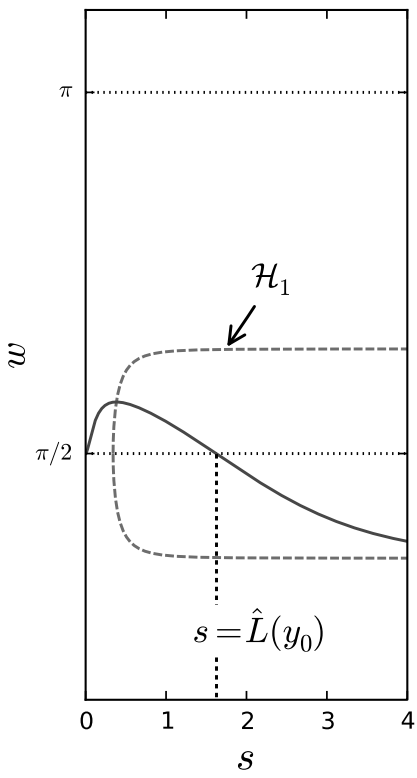

(a)

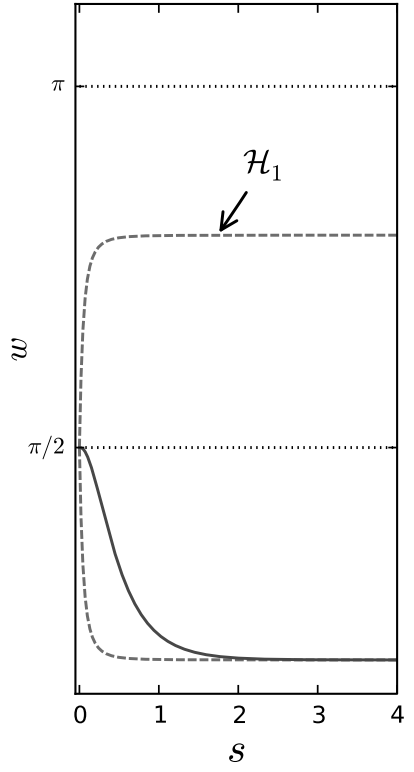

(b)

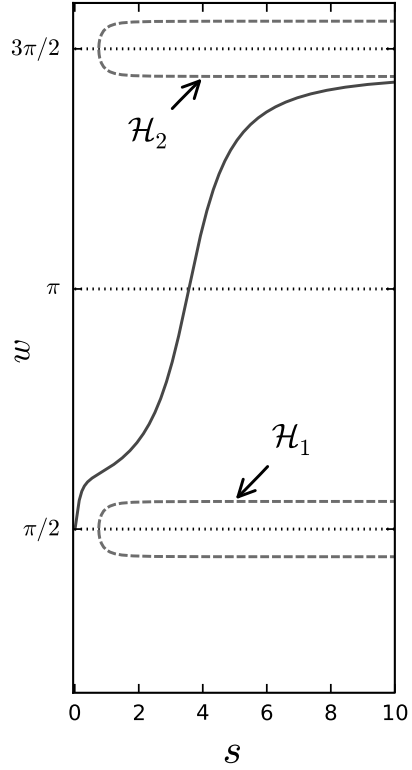

(c)

Fig. 10. Solid curves in (a) to (c) are solutions to (5.2) 1 with $w(0)=\pi / 2$ for different values of $y_{0}$. (a) $y_{0}=.2955$, (b) $y_{0}>d$, and (c) $d^{-}<y_{0}<D$.

have positive slope, so that $w$ is increasing. Conversely, inside the curve, the tangent vectors have negative slope and $w$ is decreasing.

Fig. 10 (a) also shows the solution $w$ to $(5.2)_{1}, w(0)=\pi / 2$ for $y_{0}=.2955$. The solution increases initially, until it crosses the isocline, after which it decreases to approach the lower half of $\mathcal{H}_{1}$ as $s \rightarrow \infty$. The graph of $w$ must cross the line $w=\pi / 2$ at some unique $s$ value, which we label $\widehat{L}\left(y_{0}\right)$ in the figure. As $y_{0}$ increases from the value corresponding to Fig. 10 (a), the left end of $\mathcal{H}_{1}$ moves to the left. The solution $w$ to $(5.2)_{1}, w(0)=\pi / 2$ is qualitatively the same as the solution in Fig. 10(a)-in particular, $w$ crosses the horizontal line through $\pi / 2$ at some unique value of $s-$ until, at $y_{0}=d$, the isocline $\mathcal{H}_{1}$ touches the $w$-axis at $w=\pi / 2$. For $y_{0}>d$, the solution instead decreases monotonically from $\pi / 2$ to a constant between 0 and $\pi / 2$. See Fig. 10 (b). In particular, $w$ never crosses an odd multiple of $\pi / 2$ and hence $(3.10)_{1}$ and $(3.11)_{2}$ has no non-trivial solutions for $y_{0}>d$. On the other hand, as $y_{0}$ decreases from the value corresponding to Fig. 10(a), the left end of $\mathcal{H}_{1}$ moves to the right. The solution $w$ to $(5.2)_{1}, w(0)=\pi / 2$ is qualitatively the same as the solution in Fig. 10(a) up to some critical value $D$ of $y_{0}$. For $d^{-}<y_{0}<D, w$ no longer hits $\mathcal{H}_{1}$ and instead increases and approaches the lower branch of the curve $\mathcal{H}_{2}$, another piece of the horizontal isocline. See Fig. 10(c). It is again the case that $w$ never crosses an odd multiple of $\pi / 2$. One checks numerically that $D>d^{-}$.

For $D<y_{0}<d$, we let $\widehat{L}\left(y_{0}\right)$ denote the $s$ value at which the graph of $w$ crosses the line $w=\pi / 2$. That $\widehat{L}$ depends continuously on $y_{0}$ follows from a standard theorem on the continuous dependence of a solution to an ordinary differential equation on parameters in the equation. Next, one checks that $D<y_{0}^{1}<y_{0}^{2}<d$ implies that $g\left(s, y_{0}^{1}\right)>g\left(s, y_{0}^{2}\right)$ for all $s>0$. An appropriate comparison theorem for ordinary differential equation-for example, Theorem 1.2 in Chapter 8 of Coddington and Levinson (1955)-implies that $w\left(s, y_{0}^{1}\right)>w\left(s, y_{0}^{2}\right)$ for all $s>0$, where here we show the explicit dependence of $w$ on $y_{0}$. Therefore $\widehat{L}$ is a decreasing function of $y_{0}$.

Now we define $L \rightarrow c_{0}(L)$ as the inverse of $\widehat{L}$. The observations in the previous two paragraphs imply that $c_{0}$ is decreasing as function of $L$ and that all the eigenvalues greater than $d^{-}$correspond to points on $c_{0}$ in $R_{1}$. Lastly, we note by $(5.2)_{2}$ that $r$ has no zeros if $r(0) \neq 0$. It follows that $\theta$ has no zeros if the graph of $w$ looks like the solution depicted Fig. 10(a).

\section{A.1. The Properties of the Eigencurves $c_{k}$ for $k=1,2, \ldots$}

We consider $(5.2)_{1}$ with $w(0)=\pi / 2$ for $0<y_{0}<d^{-}$. In this case, the horizontal isocline does not intersect the right half $s>0$ of the $s w$ - plane, the right-hand side of $(5.2)_{1}$ is positive for all $s>0$, and hence the solution $w$ is always increasing.

Fig. 11 depicts solutions for two different values of $y_{0}$. The graph of a typical solution $w\left(\cdot, y_{0}\right)$ crosses each of the lines $w=(2 k+1) \pi / 2$ for $k=1,2, \ldots$. The unique $s$ value of each such crossing is denoted $\widehat{L}_{k}\left(y_{0}\right)$. In Fig. 11, the first 3 crossings for each solution curve are labeled. As discussed in the previous subsection, we know that the function $\widehat{L}_{k}$ depends continuously on $y_{0}$. Next,

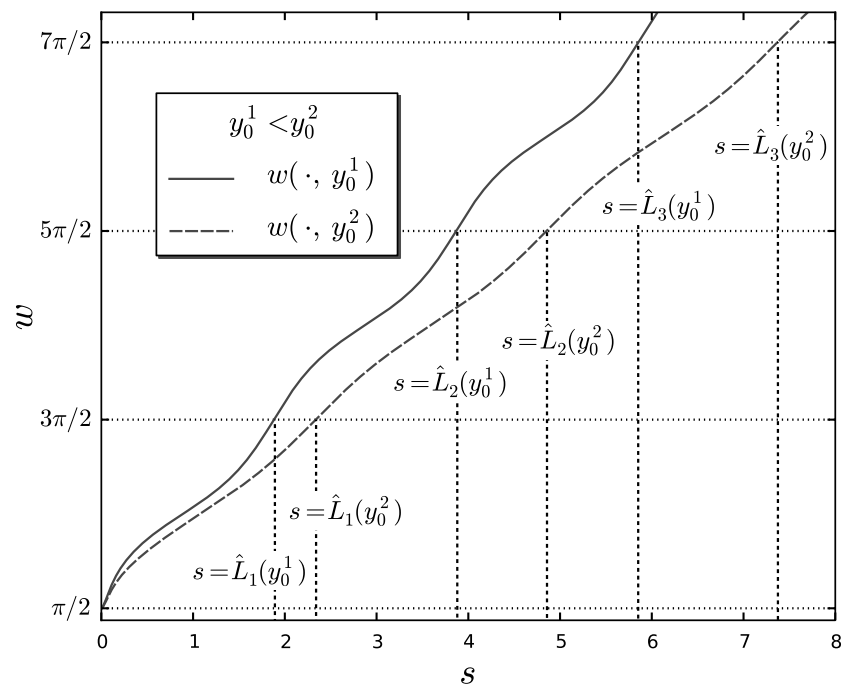

Fig. 11. Solutions to (5.2) $)_{1}$ with $w(0)=\pi / 2$ for different values of $y_{0}$. 


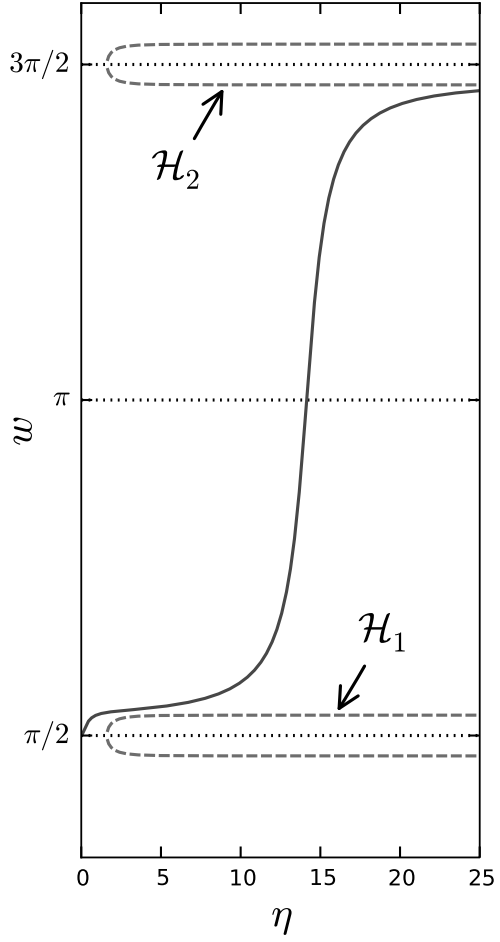

(a)

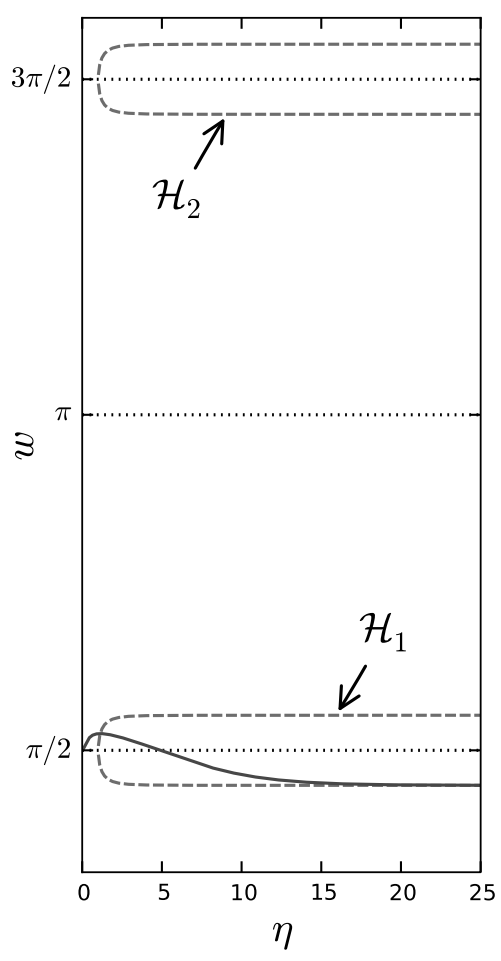

(b)

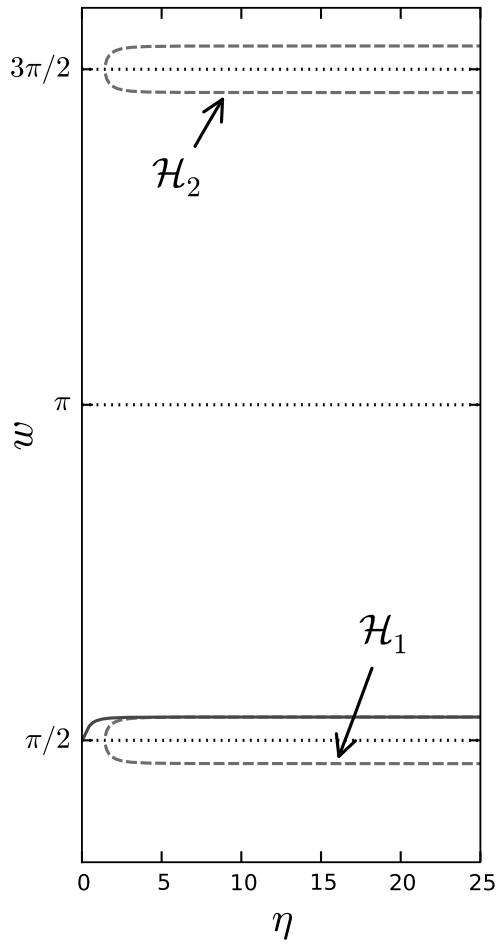

(c)

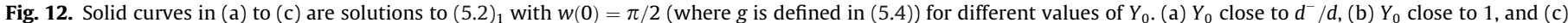
$Y_{0}=Y_{0}^{*}$.

one can check that if $0<y_{0}^{1}<y_{0}^{2}<d^{-}$, then $g\left(s, y_{0}^{1}\right)>g\left(s, y_{0}^{2}\right)$ for all $s>0$. An appropriate comparison theorem for ordinary differential equation implies that $w\left(s, y_{0}^{1}\right)>w\left(s, y_{0}^{2}\right)$ for all $s>0$, where here we show the explicit dependence of $w$ on $y_{0}$. Therefore $\widehat{L}_{k}$ is an increasing function of $y_{0}$.

Now we define $L \rightarrow c_{k}(L)$ as the inverse of $\widehat{L}_{k}$ for $k=1,2, \ldots$. The observations in the previous paragraph imply that $c_{k}$ is increasing as function of $L$ and that all the eigenvalues in $\left(0, d^{-}\right)$correspond to points on the curves $c_{k}$ in $R_{2}$. Lastly, we note by $(5.2)_{2}$ that $r$ has no zeros if $r(0) \neq 0$. By $(5.1)_{1}$, the zeros of $\theta$ are the values of $s$ at which $w$ crosses a horizontal line through a multiple of $\pi$. It follows that a point on $c_{k}$ corresponds to a solution $\theta$ of (3.10) $)_{1}$ and (3.11) $)_{2}$ with exactly $k$ zeros.

\section{A.2. The existence and uniqueness of $Y_{0}^{*}$ in Section 4}

For (4.25), we introduce the same change of variables as (5.1). Then $w, r$ satisfy (5.2) where here $g$ is defined by

$g\left(\eta, Y_{0}\right)=-\hat{\beta}^{-1} N_{0}(\eta)=\hat{\beta}^{-1} \int_{0}^{\eta} \hat{f}\left(Y_{0}+\xi\right) d \xi$.

Fig. 12 shows the solutions to $(5.2)_{1}$ with $w(0)=\pi / 2$ for three different values of $Y_{0}$. The two curves labeled $\mathcal{H}_{1}$ and $\mathcal{H}_{2}$ are subsets of the horizontal isocline for (5.2) $)_{1}$. Outside these two curves, the tangent vectors have positive slope and $w$ is increasing, while inside these two curves, the tangent vectors have negative slope and $w$ is decreasing. Fig. 12(a) shows the solution for a value of $Y_{0}$ in $\left(d^{-} / d, 1\right)$ and close to $d^{-} / d$. The solution $w$ increases initially, and this initial increase is sufficient for $w$ to pass above the curve $\mathcal{H}_{1}$, after which $w$ increases rapidly until it approaches the lower half of the curve $\mathcal{H}_{2}$. This behavior is typical for any value of $Y_{0}$ close to $d^{-} / d$. Fig. 12(b) shows the solution for $Y_{0}$ in $\left(d^{-} / d, 1\right)$ and $Y_{0}$ close to 1 . For this value of $Y_{0}$ the curve $\mathcal{H}_{1}$ is wider from top to bottom and its left endpoint has moved closer to the $w$-axis.
Although $w$ again increases initially, it fails to clear the isocline. Upon crossing $\mathcal{H}_{1}, w$ decreases and approaches the lower half of $\mathcal{H}_{1}$. This behavior is typical for any value of $Y_{0}$ close to 1 .

By a straightforward argument based on the continuous dependence of the solutions of $(5.2)_{1}$ on the parameter $Y_{0}$, on an appropriate comparison theorem (for example, Chapter 8, Theorem 1.2 in Coddington and Levinson (1955)), and on the details of the vector field, one can show that there exists a unique value $Y_{0}^{*}$ of $Y_{0}$ between $d^{-} / d$ and 1 such that the solution of $(5.2)_{1}$ with $w(0)=\pi / 2$ behaves as indicated in Fig. 12(c). In this case, $w$ approaches the upper half of $\mathcal{H}_{1}$.

In Fig. 12(a) and (b), the graph of $w$ approaches the lower half of either $\mathcal{H}_{1}$ or $\mathcal{H}_{2}$ and hence $w$ is converging to a constant that is either between $\pi$ and $3 \pi / 2$ (Fig. $12(\mathrm{a})$ ) or between 0 and $\pi / 2$ (Fig. 12(b)). In either case, $\sin w \cos w$ converges to a positive number as $\eta \rightarrow \infty$. Now we consider $(5.2)_{2}$. Noting that $g\left(\eta, Y_{0}\right)<0$ for $\eta$ sufficiently large, we see that $(1-g) \sin w \cos w \geqslant \bar{c}>0$ for $\eta$ sufficiently large for some positive constant $\bar{c}$. Hence $r \rightarrow \infty$ if $r(0)>0$ and $r \rightarrow-\infty$ if $r(0)<0$. From (5.1), it follows that in either case $\theta_{1}$ will not satisfy (4.27).

In Fig. 12(c), the graph of $w$ approaches the upper half of $\mathcal{H}_{1}$, which implies that $w$ is converging to a constant between $\pi / 2$ and $\pi$ and hence that $(1-g) \sin w \cos w \leqslant \bar{c}<0$ for $\eta$ sufficiently large for some negative constant $\bar{c}$. Hence from $(5.2)_{2}$ it follows that $r \rightarrow 0$ if $r(0) \neq 0$. Therefore (5.1) implies that $\theta_{1}$ and $\theta_{1}^{\prime}$ satisfy (4.27). We conclude that $Y_{0}^{*}$, which corresponds to Fig. 12(c), is the unique value of $Y_{0}$ for which (4.25) and (4.27) has a non-trivial solution.

\section{References}

Aliee, M., Najafi, A., 2008. Mechanical properties of an adsorbed elastic polymer in contact with a rigid membrane. Phys. Rev. E 78, 051802.

Antman, S., 2005. Nonlinear Problems of Elasticity. Applied Mathematical Sciences, vol. 107. Springer. 
Attard, P., Parker, J.L., 1992. Deformation and adhesion of elastic bodies in contact. Phys. Rev. A 46, 7959-7971.

Autumn, K., Sitti, M., Liang, Y.A., Peattie, A.M., Hansen, W.R., Sponberg, S., Kenny, T.W., Fearing, R., Israelachvili, J.N., Full, R.J., 2002. Evidence for van der waals adhesion in gecko setae. In: Proceedings of the National Academy of Sciences, vol. 99, pp. 12252-12256. <http://www.pnas.org/content/99/19/12252.full. pdf + html $>$.

Bico, J., Roman, B., Moulin, L., Boudaoud, A., 2004. Adhesion: elastocapillary coalescence in wet hair. Nature 432, 690.

Booth, T.J., Blake, P., Nair, R.R., Jiang, D., Hill, E.W., Bangert, U., Bleloch, A., Gass, M., Novoselov, K.S., Katsnelson, M.I., Geim, A.K., 2008. Macroscopic graphene membranes and their extraordinary stiffness. Nano Letters 8, 2442-2446. PMID 18593201. <http://pubs.acs.org/doi/pdf/10.1021/nl801412y>.

Bunch, J.S., van der Zande, A.M., Verbridge, S.S., Frank, I.W., Tanenbaum, D.M., Parpia, J.M., Craighead, H.G., McEuen, P.L., 2007. Electromechanical resonators from graphene sheets. Science 315, 490-493 <http://www.sciencemag.org/ content/315/5811/490.full.pdf $>$.

Caflisch, R.E., Maddocks, J.H., 1984. Nonlinear dynamical theory of the elastica. Proc. R. Soc. Edin Section: A Math. 99, 1-23.

Coddington, E., Levinson, N., 1955. Theory of Ordinary Differential Equations. McGraw-Hill, New York.

Doedel, E., Champneys, A.R., Fairgrieve, T., Kuznetsov, Y., Oldeman, B., Paffenroth, R., Sandstede, B., Wang, X., C., Z., 2006. AUTO-07P: continuation and bifurcation software for ordinary differential equations. Technical Report. Concordia University. <http://indy.cs.concordia.ca/auto/>.

Evans, A.A., Lauga, E., 2009. Adhesion transition of flexible sheets. Phys. Rev. E 79, 066116.

Geim, A.K., 2009. Graphene: status and prospects. Science 324, 1530-1534<http:// www.sciencemag.org/content/324/5934/1530.full.pdf>.

Girifalco, L.A., Lad, R.A., 1956. Energy of cohesion, compressibility, and the potential energy functions of graphite system. J. Chem. Phys. 25, 693-697.

Jia, X., Yang, J., Kitipornchai, S., 2011. Pull-in instability of geometrically nonlinear micro-switches under electrostatic and casimir forces. Acta Mech. 218, 161174. http://dx.doi.org/10.1007/s00707-010-0412-8.

Kim, Eun-Ah, Castro Neto, A.H., 2008. Graphene as an electronic membrane. EPL 84, 57007.

Lee, C., Wei, X., Kysar, J.W., Hone, J., 2008. Measurement of the elastic properties and intrinsic strength of monolayer graphene. Science 321, 385-388 http:// www.sciencemag.org/content/321/5887/385.full.pdf.

Li, C., Chou, T.W., 2003. A structural mechanics approach for the analysis of carbon nanotubes. Int. J. Solids nd Struct. 40, 2487-2499.

Liao, M., Koide, Y., 2011. Carbon-based materials: growth, properties, mems/nems technologies, and mem/nem switches. Crit. Rev. Solid State Mater. Sci. 36, 66101 <http://www.tandfonline.com/doi/pdf/10.1080/10408436.2011.572748>.

Lu, Q., Huang, R., 2009. Nonlinear mechanics of single-atomic-layer graphene sheets. Int. J. Appl. Mech. 1, 443-467.

Lu, Q., Huang, R., 2010. Excess energy and deformation along free edges of graphene nanoribbons. Phys. Rev. B 81, 155410.

Majidi, C.S., Groff, R.E., Fearing, R.S., 2005. Attachment of fiber array adhesive through side contact. J. Appl. Phys. 98, 103521.

Novoselov, K.S., Geim, A.K., Morozov, S.V., Jiang, D., Zhang, Y., Dubonos, S.V., Grigorieva, I.V., Firsov, A.A., 2004. Electric field effect in atomically thin carbon films. Science 306, 666-669 <http://www.sciencemag.org/cgi/reprint/306/ 5696/666.pdf>.

Novoselov, K., Jiang, D., Schedin, F., Booth, T., Khotkevich, V., Morozov, S., Geim, A. 2005. Two-dimensional atomic crystals. Proc. Natl. Acad. Sci. 102, 1045110453.

Oyharcabal, X., Frisch, T., 2005. Peeling off an elastica from a smooth attractive substrate. Phys. Rev. E 71, 036611.

Peng, J., Wu, J., Hwang, K., Song, J., Huang, Y., 2008. Can a single-wall carbon nanotube be modeled as a thin shell? J. Mech. Phys. Solids 56, 2213-2224.
Pradhan, S.C., Murmu, T., 2009. Small scale effect on the buckling of single-layered graphene sheets under biaxial compression via nonlocal continuum mechanics. Comput. Mater. Sci. 47, 268-274. http://dx.doi.org/10.1016/j.commatsci.2009. 08.001.

Pradhan, S., Murmu, T., 2010. Small scale effect on the buckling analysis of singlelayered graphene sheet embedded in an elastic medium based on nonlocal plate theory. Phys. E: Low-dimensional Syst. Nanostruct. 42, 1293-1301.

Purohit, P.K., Kondev, J., Phillips, R., 2003. Mechanics of dna packaging in viruses Proc. Natl. Acad. Sci. 100, 3173-3178 http://www.pnas.org/content/100/6 3173.full.pdf+html.

Reddy, C.D., Ramasubramaniam, A., Shenoy, V.B., Zhang, Y.W., 2009. Edge elastic properties of defect-free single-layer graphene sheets. Appl. Phys. Lett. 94 101904.

Reddy, C.D., Zhang, Y.W., Shenoy, V.B., 2011. Influence of substrate on edge rippling in graphene sheets. Model. Simul. Mater. Sci. Eng. 19, 054007.

Rief, M., Clausen-Schaumann, H., Gaub, H.E., 1999. Sequence-dependent mechanics of single dna molecules. Nat. Struct. Mol. Biol. 6, 346-349.

Robertson, D.H., Brenner, D.W., Mintmire, J.W., 1992. Energetics of nanoscale graphitic tubules. Phys. Rev. B 45, 12592-12595.

Sabio, J., Seoánez, C., Fratini, S., Guinea, F., Neto, A.H.C., Sols, F., 2008. Electrostatic interactions between graphene layers and their environment. Phys. Rev. B (Condens. Matter Mater. Phys.) 77, 195409.

Sakhaee-Pour, A., 2009. Elastic buckling of single-layered graphene sheet. Comput. Mater. Sci. 45, 266-270.

Schniepp, H., Li, J.L., McAllister, M., Sai, H., Herrera-Alonso, M., Adamson, D. Prud'homme, R., Car, R., Saville, D., Aksay, I., 2006. Functionalized single graphene sheets derived from splitting graphite oxide. J. Phys. Chem. B 110 8535-8539.

Smith, D.E., Tans, S.J., Smith, S.B., Grimes, S., Anderson, D.L., Bustamante, C., 2001 The bacteriophage [phis]29 portal motor can package dna against a large internal force. Nature 413, 748-752.

Strus, M.C., Raman, A., 2009. Identification of multiple oscillation states of carbon nanotube tipped cantilevers interacting with surfaces in dynamic atomic force microscopy. Phys. Rev. B 80, 224105.

Strus, M.C., Zalamea, L., Raman, A., Pipes, R.B., Nguyen, C.V., Stach, E.A., 2008. Peeling force spectroscopy: exposing the adhesive nanomechanics of one-dimensional nanostructures. Nano Letters 8, 544-550. PMID: 18189440. <http:// pubs.acs.org/doi/pdf/10.1021/nl0728118>.

Suk, J.W., Piner, R.D., An, J., Ruoff, R.S., 2010. Mechanical properties of monolayer graphene oxide. ACS Nano. 4, 6557-6564 <http://pubs.acs.org/doi/pdf/10.1021/ nn101781v>.

Tersoff, J., 1992. Energies of fullerenes. Phys. Rev. B 46, 15546-15549.

Tu, Z.c., Ou-Yang, Z.c., 2002. Single-walled and multiwalled carbon nanotubes viewed as elastic tubes with the effective young's moduli dependent on layer number. Phys. Rev. B 65, 233407.

Wilber, J., 2010. Buckling of graphene layers supported by rigid substrates. J. Comput. Theor. Nanosci. 7, 2338-2348.

Wilber, J., Buldum, A., Clemons, C., Quinn, D., Young, G., 2007a. Continuum and atomistic modeling of interacting graphene layers. Phys. Rev. B 75, 045418.

Wilber, J.P., Clemons, C.B., Young, G.W., Buldum, A., Quinn, D.D., 2007b. Continuum and atomistic modeling of interacting graphene layers. Phys. Rev. B 75, 1-10.

Wu, Y.H., Yu, T., Shen, Z.X., 2010. Two-dimensional carbon nanostructures: fundamental properties, synthesis, characterization, and potential applications. J. Appl. Phys. 108, 071301.

Yakobson, B., Brabec, C., Bernholc, J., 1996. Nanomechanics of carbon nanotubes: instabilities beyond the linear response. Phys. Rev. Lett. 76, 2511-2514.

Yap, H.W., Lakes, R.S., Carpick, R.W., 2007. Mechanical instabilities of individual multiwalled carbon nanotubes under cyclic axial compression. Nano Lett. 7 , 1149-1154 http://pubs.acs.org/doi/pdf/10.1021/nl062763b.

Zhao, Y., Wang, L., Yu, T., 2003. Mechanics of adhesion in memsa review. J. Adhesion Sci. Technol. 17, 519-546. 